\title{
Distribution path optimization of automated warehouse based on polynomial time algorithm
}

\author{
Lvqing Yang ${ }^{1, a}$, Wei-wei LIU ${ }^{2, b,}$, Youjing Bai ${ }^{1, c}$ \\ ${ }^{1}$ Software School,Xiamen University, Xiamen 361005,China \\ ${ }^{2}$ Faculty of Informatics, Fujian Vocational College of Agriculture, Fuzhou, Fujian 350001, China

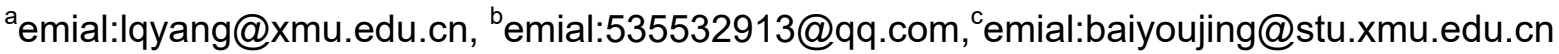 \\ ${ }^{*}$ Corresponding author
}

Keywords: Order picking, Path optimization, Polynomial time algorithm, Rectilinear norm movement mode.

Abstract. This paper discusses the use of polynomial time algorithm for solving the distribution path optimization of unit load automatic distribution system which was used in storage systems commonly, under the condition of specified goods consolidation and rectilinear norm movement mode, establishes the mathematical models according to the characteristics, uses the main circuit which is formed by the algorithm's directed graph as the optimal solution of the model problem, the time complexity of the algorithm is $O(n)$, so it has great practical value.

\section{Introduction}

The goods homework also called picking is the activities of Warehousing and distribution center remove goods from the goods shelves according to customer orders under the name of goods, quantity and storage, and put in a specified location, complete user requirements of distribution, its purpose is to set the required goods correctly and quickly. So the research and application of distribution operation's operation method has a strong practical significance to improve storage efficiency.

Many factors affect the efficiency of distribution work, can be divided into external factors and internal factors, the former mainly includes market channels, customer demand mode, supplier replenishment mode and so on, the latter mainly refers to the configuration of the distribution center, including the distribution and storage strategy of distribution system, the choice of distribution operation method and so on. There are four major categories of distribution system according to the different choice of shelf and picking equipment and other hardware facilities [1]: the traditional 
rectangular distribution system, $\mathrm{MOB}$ distribution system which staff and distribution equipment cooperate with each other, unit load automatic distribution system which sorting and storage operations cross and carousel distribution system. In recent years, the research mainly includes[2]: the optimization problem of the fixed goods shelf picking up is TSP (traveling salesman problem), finding the optimal or sub optimal path by using the heuristic rule, the maximal algebra method and the improved Hopfield neural network method; the optimization problem of the access path of the single servo layered horizontal rotating shelf system by using the improved simulated annealing algorithm; the optimization problem of the access path of the double servo layered horizontal rotating shelf system by using genetic algorithm. This paper mainly discusses the distribution path optimization of unit load automatic distribution system which was used in storage systems commonly, under the condition of specified goods consolidation and rectilinear norm movement mode, and uses polynomial time algorithm to solve the problem.

\section{Problem description and model establishment}

The optimization of path or sequence can help the picking person obtain optimal or satisfactory goods distribution order, then save the picking time, shorten the picking distance[3]. The problem can be described as follows:

Known: (1)The specific location of the sorting system

(2)A set of selected items and the storage location of the selected areas

(3)Collection point location, centralized or decentralized, fixed or variable

Constraint: (1)Order picking personnel path movement, it can be a straight line, the European movement mode and so on.

(2)Picking method, a pick up all the items returned, picking the next item after picking a set of goods returned

Target: picking the shortest time or minimum path

Assume that picking task is chosen from sorting area $n$ class items $A_{1}, A_{2}, \ldots, A_{n}$, these items are stored separately in the sorting area $P_{1}, P_{2}, \ldots, P_{n}$, and the set point of the goods are $\bar{P}_{1}, \bar{P}_{2}, \ldots, \bar{P}_{n}$, the location parameter is known. Assume that the $A_{i}$ item is specified at the point of collection point $\bar{P}_{i}$, taking the direction of the truck as the $\mathrm{X}$ axis, the anchor point of the picking equipment is set up to establish a rectangular coordinate system, get the position coordinates : coordinate of storage location $P_{j}$ in sorting area are $\left(x_{j}, y_{j}\right), j=1,2 \ldots, \mathrm{n}$; coordinates of set point $\bar{P}_{i}$ are $\left(\bar{x}_{i}, 0\right), \mathrm{i}=1,2, \ldots, \mathrm{n}$; coordinates of the anchor point $\operatorname{are}\left(\bar{x}_{0}=x_{0}=0, \mathrm{y}_{0}=0\right)[4]$.

Picking equipment choose goods $A_{i}$ after picking goods $A_{i}$, according to rectilinear norm movement mode, the mobile route of the picking device includes the path from the point of collection $\bar{P}_{i}$ to the storage location $P_{i}$ for loading $A_{i}$ which is represent as $\left|\bar{x}_{i}-x_{j}\right|+y_{j}$, and the path from the storage location $P_{i}$ to the point of collection $\bar{P}_{i}$ for transporting goods $A_{i}$ which is represent as $\left|x_{j}-\bar{x}_{j}\right|+y_{j}$ total as $+\left|\bar{x}_{i}-x_{i}\right|+2 y_{\mathrm{j}}$. Introduce variable $Z_{i i}$, supplement $A_{i}$ after $A_{i}$, when $i \neq j, Z_{i j}=1(\mathrm{j}=0,1,2, \ldots, \mathrm{n} ; \mathrm{i}=0,1,2, \ldots, \mathrm{n})$, otherwise $Z_{i j}=0$. Set the total path of picking equipment to complete all picking operations return to the starting point as $\left|x_{j}-\bar{x}_{j}\right|$ 


$$
\mathrm{F}=\sum_{\substack{i=0 \\ i \neq j}}^{n} \sum_{j=0}^{n}\left\{\left|x_{j}-\bar{x}_{j}\right|+\left|\bar{x}_{i}-x_{j}\right|+2 y_{j}\right\}, Z_{i i}=\sum_{\substack{i=0 \\ i \neq j}}^{n} \sum_{j=0}^{n}\left\{\left|x_{j}-\bar{x}_{j}\right|+\left|\bar{x}_{i}-x_{j}\right|\right\} \bullet Z_{i j}+2 \sum_{i=0}^{n} y_{i}
$$

Where $2 \sum_{i=0}^{n} y_{i}$ is a fixed value, let $\bar{F} \sum_{\frac{i=0}{i \neq j}} \sum_{j=0}^{n}\left\{\left|x_{j}-\bar{x}_{j}\right|+\left|\bar{x}_{i}-x_{j}\right|\right\} \bullet Z_{i j}[5]$. Set up an integer
programming model:

$$
\begin{aligned}
& \operatorname{Min} \bar{F} \\
& s \bullet t \bullet\left\{\begin{array}{l}
\sum_{\frac{i=0}{i \neq j}}^{n} Z_{i j}=1, i=0,2, \ldots, n \\
\sum_{i=0}^{n} Z_{i j}=1, j=0,1, \ldots, n \\
\sum_{i \neq j}^{j \neq i ; i, j \in S} Z_{i j} \leq|S|-1, \forall S \subset\{0,1,2, \ldots, n\}
\end{array}\right. \\
& Z_{i j} \in\{1,0\}, i \neq j
\end{aligned}
$$

$|\mathrm{S}|$ represents the number of elements in the set S; Formula (1) is the goal to achieve; the constraints (2) and (3) guarantee the picking for each distribution goods occurs only once; formula (4) can effectively prevent picking equipment from picking up all items before returning to the starting point; formula (5) indicates that the decision variables are only 0 or 1 .The model includes $\mathrm{n}^{2}$ two element decision variables and $2^{\mathrm{n}+1}+\mathrm{n}-2$ constraints[6].

\section{Problem solving}

The time complexity of the general algorithm to solve the integral linear programming is the exponential function of the scale of the problem, the computing time is too large for large scale problems, so polynomial time algorithm is used to solve the problem[7].

Firstly, all the storage sites are mapped to the corresponding point $p_{j}(\mathrm{j}=0,1, \ldots, \mathrm{m})$ on the $\mathrm{X}$ axis according to the horizontal coordinate $x_{k}(\mathrm{k}=0,1, \ldots, \mathrm{n})$, and $\mathrm{p}_{\mathrm{m}}=\max \left\{p_{j}\right\}, \mathrm{P}_{0}$ is the starting point for picking goods, the moving path of $A_{j}$ replenishment operation corresponding to the direction arc from $\mathrm{X}$ axis point of coordinate $x_{j}$ to point of coordinate $\bar{x}_{j}$, its weight is the $\left|x_{j}-\bar{x}_{j}\right|$ part of the objective function $\bar{F}$, there are total $\mathrm{n}$ of the directed arc, and the value is fixed which has nothing to do with decision variables, use the solid line to express on the graph; the mobile path of return to load $A_{j}$ after picking out $A_{i}$ mapped to the direction arc from point of coordinate $\bar{x}_{i}$ to point of coordinate, its weight is the $\left|\bar{x}_{i}-x_{j}\right|$ part of the objective function $\bar{F}$, the value varies with the decision variable, use the dotted line $E_{j}$ to express on the graph. Make express $X$ axis line between two consecutive points $p_{j-1}$ and $p_{j}$, the value is the distance between two points $\left|p_{j-1} p_{j}\right|$ [8].

According to the constraint condition, the model can be converted to[9]: increase the number of the imaginary arc properly in the directed graph which has $\mathrm{m}$ points $p_{j}$ and $\mathrm{n}$ fixed real directed 
$\operatorname{arc}(\mathrm{j}=0,1, \ldots, \mathrm{m})$, connect real directed arc to construct the shortest directed European circuit which through all fixed directed arc only once, the value of the directed arc is the distance of the through line segment. Define any path of starting and terminating at the same point as the circuit; the circuit which contain all fixed (real) directed arc is the complete circuit; the directed circuit which start from the point $p_{0}$ and return to point $p_{0}$ is the main circuit; the directed arcs of the same direction of the $\mathrm{X}$ axis as the forward arcs, on the contrary is the back arc. According to the nature of the directed Euler circuit: in the complete circuit, the numbers of forward and backward arcs which through any line segment are equal. So the essence of solving the model problem is minimizing the sum of weights of the other directed arcs which add on the original direction graph $\mathrm{x}$.

Design algorithm I [1]: connect all fixed directed arcs, calculate the number of forward and backward $\operatorname{arcs} n_{j 1}$ and $n_{j 2}$ by any line segment $E_{j}$.If $n_{j 1}=n_{j 2}=0$, adding a forward and backward arc to the line segment $E_{j}$; if $n_{j 1} \neq n_{j 2}$, adding the number of $\left|n_{j 1}-n_{j 2}\right|$ dotted directed arc to the line segment $E_{j}$. The directed graph constructed by this algorithm is the optimal solution of the model problem after the relaxation of the constraint condition (4); if the main circuit is the complete circuit, the directed graph is the optimal solution of the problem model.

But in general, the main circuit does not include all fixed (real) directed arcs, such as the main circuit separates with the two parallel sub circuits, the main circuit separates with the series ion circuit, see figure 1,2 below:

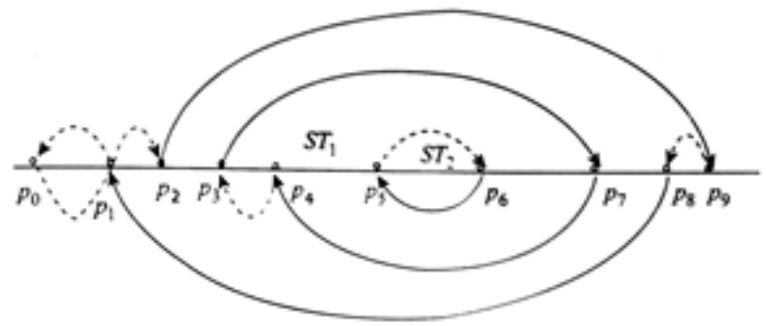

Figure 1 Main circuit separates with the two parallel sub circuit

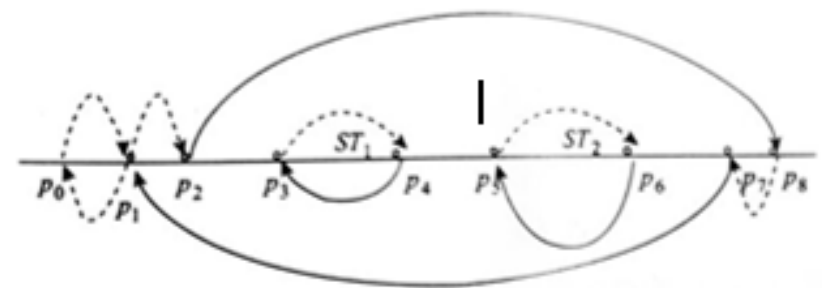

Figure 2 Main circuit separates with the series ion circuit

So design the algorithm II[1], construct a new directed arc connecting these sub circuits to the main circuit, make the main circuit to form a complete circuit. The algorithm idea is: how to use the new directed arc of shortest weights to connect these separated sub circuit to form the needed complete circuit.

(1)Let $\mathrm{j}=0$ 
(2)If $p_{j}$ is on the main circuit, jump to step (7)

(3)Let $\mathrm{k}=1$

(4)Find the ion circuit $S T_{k}$, let $S_{k}=p_{j}, s_{k}=p_{j-1} ; T_{k}=p_{e}, t_{k}=p_{e+1}$, Which $p_{e}$ represents the maximum point in the sub circuit $S T_{i}$. If $t_{i}$ is not on the main circuit, then $\mathrm{k}=\mathrm{k}+1$, jump to step (4)

(5)If $\mathrm{k}=1$, and if $\left|S_{k} S_{k}\right| \leq\left|T_{k} t_{k}\right|$, construct two opposite directional arc to connect points $S_{k}$ and $s_{k}$, otherwise connect points $T_{k}$ and $t_{k}$

(6)Let $w_{0}=\left|S_{1} S_{1}\right|, w_{i}=\left|S_{i+1} T_{i}\right| \mathrm{i}=1,2, \ldots, \mathrm{k}-1, \quad w_{k}=\left|T_{k} t_{k}\right|, \quad w_{i}=\max \left\{w_{i} \mid i=0,1, \ldots, k-1\right\}$

(1) If $w_{k}>\max \left\{w_{0}, w_{i *}\right\}$, construct pair of opposite directed arc to connect points $s_{1}$ and $S_{1}, \quad T_{i}$ and $S_{i+1}, \quad \mathrm{i}=1,2, \ldots, \mathrm{K}-1$

(2)If $w_{0}>\max \left\{w_{k}, w_{i *}\right\}$, construct pair of opposite directed arc to connect points $T_{i}$ and $S_{i+1}$, $T_{k}$ and $t_{k}, \mathrm{i}=1,2, \ldots, \mathrm{k}-1$,

(3) If $w_{i *}>\max \left\{w_{0}, w_{k}\right\}$, construct pair of opposite directed arc to connect points $s_{1}$ and $S_{1}, T_{i}$ and $S_{i+1}, \mathrm{i}=1,2, \ldots, i^{*}-1$, as well as points $T_{k}$ and $t_{k}, T_{i}$ and $S_{i+1}, \mathrm{i}=i^{*}+1, i^{*}+2, \ldots, \mathrm{K}-1$

(7)Let $\mathrm{j}=\mathrm{j}+1$, if $\mathrm{j} \neq \mathrm{m}$, jump to step (2); otherwise terminate the process, output the main circuit

According to formula (2) and the construction method of directed arc of the main circuit connected to the ion circuit in algorithm II, can obtain that the main circuit is the optimal solution of the model problem, the main circuit is formed of the directed graph which constructed by algorithm I and algorithm II . Finally, according to the determined main circuit of the directed graph reflects to the actual corresponding storage site, obtain the order of picking goods of the directional picking operation problem, then solve the problem[10]. The time complexity of the algorithm I and II are all $O(n)$.

\section{Trial runs}

Distribution operations of a large distribution center required choosing six kinds of goods, the location of the collection point and picking area of the six kinds of goods is shown in figure 3 below. According to the requirement of distribution center allocation, arrangement the goods distribution order, make the picking personnel or equipment to move the shortest path. (Unit: 10 meters) 


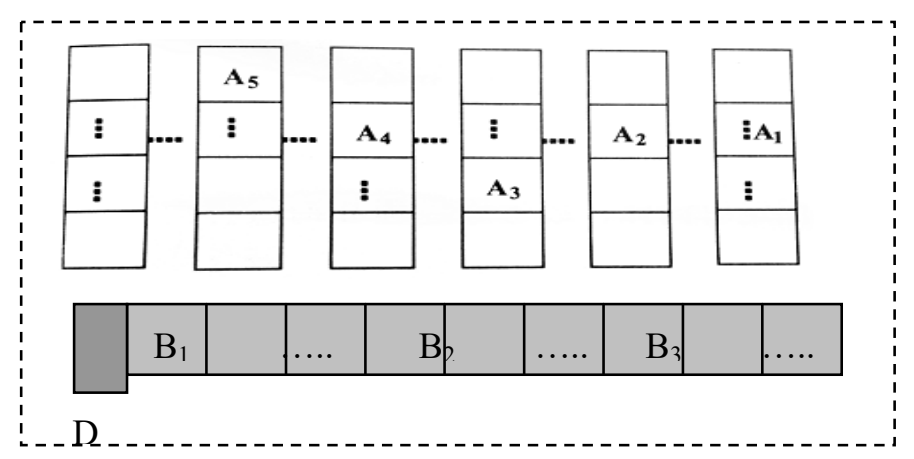

Figure 3 Distribution system of a distribution center

Firstly, determined the coordinate of the devices starting point and the each reservoir site are[11]: $\mathrm{D}(0,0) ; \mathrm{A}_{1}(16,8), \mathrm{A}_{2}(12,8), \mathrm{A}_{3}(9,6), \mathrm{A}_{4}(7,8), \mathrm{A}_{5}(3,10) ; \mathrm{B}_{1}(1,0), \mathrm{B}_{2}(5,0), \mathrm{B}_{3}(8,0), \mathrm{B}_{4}(12,0)$, $\mathrm{B}_{5}(18,0)$.The points were respectively mapped to 10 points on the $\mathrm{X}$ axis $P_{i}(\mathrm{j}=1,2,3, \ldots, 10), \mathrm{D}->\mathrm{p}_{0}, \mathrm{~B}_{1}->\mathrm{p}_{1}, \mathrm{~B}_{2^{-}}>\mathrm{p}_{3}, \mathrm{~A}_{4}->\mathrm{p}_{4}, \mathrm{~B}_{3}->\mathrm{p}_{5}, \mathrm{~A}_{3}->\mathrm{p}_{6}, \mathrm{~B}_{4}->\mathrm{p}_{7}, \mathrm{~A}_{1}->\mathrm{p}_{8}, \mathrm{~B}_{5}->\mathrm{p}_{9}$. Constructed the fixed directed arcs (The solid line), constructed other directed arc according to the algorithm I (the dotted line), Can get a directed graph as shown in figure 4:

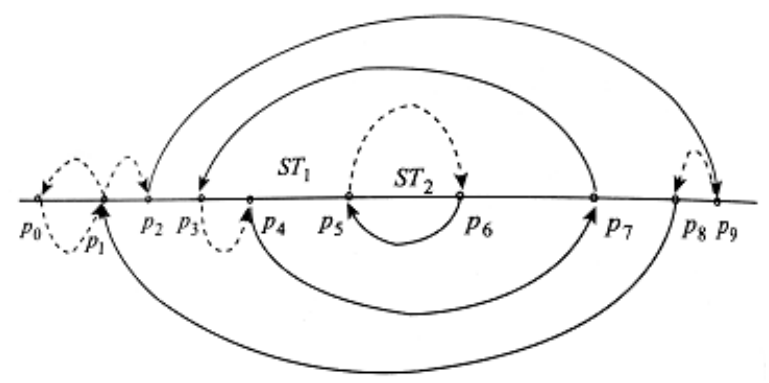

Figure 4 Initial path graph

A separate sub circuit exists in the graph, the directed main circuit did not contain all of the fixed directed arcs, so the algorithm II was used to construct directed arc to the two separated

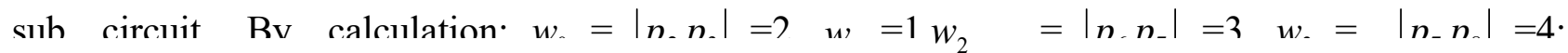
$w_{2}=\max \left\{w_{i} \mid \mathrm{i}=0,1,2\right\}$, constructed pairs of opposite directed arc of point $\mathrm{p}_{2}$ and $\mathrm{p}_{3}, \mathrm{p}_{4}$ and $\mathrm{p}_{5}, \mathrm{p}_{6}$ and $\mathrm{p}_{7}$ as shown in figure 5, then form the directed graph main which main circuit includes all fixed directed arcs. From Figure 5 the main circuit (the optimal directed Euler circuit) was: $\mathrm{p}_{0^{-}}>\mathrm{p}_{6^{-}}>\mathrm{p}_{5}->\mathrm{p}_{4}->\mathrm{p}_{7}->\mathrm{p}_{3^{-}}>\mathrm{p}_{2^{-}}>\mathrm{p}_{9^{-}}>\mathrm{p}_{8^{-}}>\mathrm{p}_{1^{-}}>\mathrm{p}_{0}$, reflected back to the corresponding storage sites, can get the order picking route : $\mathrm{D}->\mathrm{A}_{3}->\mathrm{B}_{3}->\mathrm{A}_{4}->\mathrm{B}_{4}->\mathrm{A}_{2}->\mathrm{B} 2->A 5->\mathrm{B} 5->A 1->\mathrm{B} 1->\mathrm{D}$, the total path to move of picking equipment was 1380 meters.

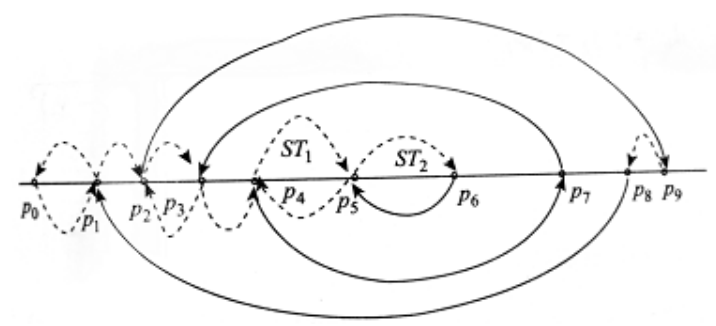

Figure 5 Optimized path graph 


\section{Conclusions}

This paper study the use of polynomial time algorithm for solving the distribution path optimization of unit load automatic distribution system, under the condition of specified goods consolidation and rectilinear norm movement mode, The main circuit formed by the directed graph of the algorithm is the optimal solution of the model problem, the time complexity of the algorithm are all $O(n)$, the computation time is very short, and has great practical value.

\section{References}

[1]Xiongzhi Wang. Study on order picking method in distribution center[M].BeiJing: China Economic Publishing House, 2008: 56-58.

[2]Guo-hui Tian, Changyou Liu. Research and development on optimization and scheduling problems in an automated warehouse [J].Journal of Shandong University of Technology, 2001(2):12-17.

[3]Xiangling $\mathrm{Xu}$, Weiping $\mathrm{Fu}$, Dexin Li et al. Study on loading/unloading scheduling of high rack warehouse based on ES [J].Logistics Technology, 2005(2):38-41.

[4] Faliang Chang, Zengxiao Liu, Zheng Xin et al. Research on the order picking optimization problem of the automated warehouse [J]. Systems Engineering Theory \& Practice, 2007(2):139-143.

[5]Yifei Chen, Jun Liu. Design and application of VRP model for storage picking operation path [J]. Computer engineering and Applications, 2006(6): 209-214.

[6]Xiaoping Zhang et al. Modern production logistics and simulation [M].Beijing: Tsinghua university press, 2000.

[7] RD Koster, T Le -Duc, KJ Roodbergen. Design and Control of Warehouse Order Picking: a literature review [J]. European Journal of Operational Research, 2007, 18(12):481-501.

[8]Zengxiao Liu, Zhanying Feng, Jian Wu et al. A simple optimization algorithm for the stacker working path of the picking - type automatic warehouse $[\mathrm{J}]$. Hoisting and conveying machinery, 2006(8): 49-51.

[9] Rana,Krishan.Order picking in narrow-aisle warehouses [J]. International Journal of Physical Distribution \&Logistics Management, 1990, 20(2):9-15.

[10]Lilin Jiang, Chi Zhang. Optimization of picking route for the automated warehouse stacker based on graph theory [J]. Logistics technology, 2010, 4(7):97-99.

[11]Xiaohui Zhao, Yingping Zheng, Lingqun Wang et al. Optimal design of picking system for high level of distribution center [J]. Logistics technology, 2007,26(1):99-111. 